\title{
Twisted Group Algebras II
}

\author{
C. M. Edwards and J. T. Lewis \\ The Queen's College, Oxford \\ Brasenose College, Oxford
}

Received January 10, 1969

\begin{abstract}
WENDEL showed that norm non-increasing isomorphisms between the group algebras of locally compact groups could be expressed in terms of group characters and topological isomorphisms. His results are extended to twisted group algebras. In particular, by applying a generalisation of WENDEL's main result to twisted group algebras over the same group, it is shown that the number of such algebras is equal to the number of orbits in a 2 -cohomology group over $G$ under the action of the automorphism group of $G$. An application to the twisted group algebra defined by WEYL's form of the canonical commutation relations is considered.
\end{abstract}

\section{$\S 1$. Indroduction}

In a previous paper [4] the properties of twisted group algebras over a separable locally compact group $G$ were studied. Many of these properties generalised those of the group algebra $L_{1}(G)$ of $G$. WENDEL [8] used the theory of centralisers to study norm non-increasing isomorphisms between two group algebras $L_{1}(G), L_{1}\left(G^{\prime}\right)$ and showed that these could be described in terms of a character of $G$ and a topological isomorphism between $G$ and $G^{\prime}$. In this paper WENDEL's results are generalised to twisted group algebras with continuous multipliers. In particular, by studying the form of norm non-increasing isomorphisms between twisted group algebras of the same group $G$, it is shown that the number, up to isometric *-isomorphism, of twisted group algebras of $G$ with continuous multipliers is equal to the number of orbits in a certain 2-cohomology group over $G$ under the action of the automorphism group of $G$.

The final section is devoted to the study of an example which is of some relevance to the quantum theory of a system with one degree of freedom.

\section{\$ 2. Notation and Basic Results}

Let $G$ be a separable locally compact group with unit element $e$ and let $m$ be a left-invariant Haar measure on $G$; let $\delta$ be the modular function on $G$; let $L_{1}(G)$ be the space of equivalence classes of complexvalued, $m$-measurable, absolutely integrable functions on $G . L_{1}(G)$ is a Banach space with respect to the norm $\|\cdot\|_{1}$ defined for each element $\psi$ of $L_{1}(G)$ by

$$
\|\psi\|_{1}=\int_{G}|\psi(x)| d m(x) .
$$


Let $T$ be the group of complex numbers of unit modulus. A continuous function $\varrho$ from $G$ to $T$ such that $\varrho(e)=1$ is said to be a 1 -cochain and a continuous function $\omega$ from $G \times G$ to $T$ is said to be a 2 -cocycle if for all $x, y, z$ in $G$,

$$
\begin{aligned}
\omega(e, x) & =\omega(x, e)=1, \\
\omega(y, z) \omega(x, y z) & =\omega(x, y) \omega(x y, z) .
\end{aligned}
$$

Let $Z^{1}(G, T), Z^{2}(G, T)$ be the sets of 1-cochains, 2-cocycles respectively. These are abelian groups when addition is defined for pairs $\varrho_{1}, \varrho_{2}$ in $Z^{1}(G, T)$ and $\omega_{1}, \omega_{2}$ in $Z^{2}(G, T)$ by

$$
\begin{aligned}
\left(\varrho_{1}+\varrho_{2}\right)(x) & =\varrho_{1}(x) \varrho_{2}(x), \\
\left(\omega_{1}+\omega_{2}\right)(x, y) & =\omega_{1}(x, y) \omega_{2}(x, y),
\end{aligned}
$$

for each pair $x, y$ of elements of $G$. For each element $\varrho$ of $Z^{1}(G, T)$ let $\partial \varrho$ be the function defined for each pair $x, y$ of elements of $G$ by

$$
(\partial \varrho)(x, y)=\varrho(x) \varrho(y) \varrho(x y)^{-1} \text {. }
$$

Then $\partial$ is a homomorphism from $Z^{1}(G, T)$ onto a subgroup $B^{2}(G, T)$ of $Z^{2}(G, T)$. The quotient group $H^{2}(G, T)=Z^{2}(G, T) / B^{2}(G, T)$ is said to be the 2-cohomology group.

The results of [4] show that to each element $\omega$ of $Z^{2}(G, T)$ there exists a multiplication $\psi_{1}, \psi_{2} \mapsto \psi_{1} \omega \psi_{2}$ and an involution $\psi \mapsto \psi^{\omega}$ on $L_{1}(G)$ defined for each element $x$ of $G$ by

$$
\begin{aligned}
\left(\psi_{1} \omega \psi_{2}\right)(x) & =\int_{G} \psi_{1}(y) \psi_{2}\left(y^{-1} x\right) \omega\left(y, y^{-1} x\right) d m(y), \\
\psi^{\omega}(x) & =\overline{\psi\left(x^{-1}\right)} \delta\left(x^{-1}\right) \overline{\omega\left(x, x^{-1}\right)}
\end{aligned}
$$

with respect to which $L_{1}(G)$ is a Banach $*$-algebra $\left(L_{1}(G), \omega\right)$, which is said to be the twisted group algebra of $G$ corresponding to the multiplier $\omega$.

A right centaliser $V$ on a Banach *-algebra $\mathfrak{U}$ is a bounded linear operator from $\mathfrak{U}$ to itself such that for each pair $a, b$ of elements of $\mathfrak{U}$

$$
V(a b)=V(a) b .
$$

Left-centalisers are similarly defined. The set $R(\mathfrak{U})$ of right centalisers on $\mathfrak{U}$ is a closed subspace of the Banach space $B(\mathfrak{U})$ of bounded linear operators from $\mathfrak{U}$ to itself.

Let $M(G)$ be the set of regular complex measures on $G$ of bounded total variation. $M(G)$ is a Banach space with respect to the norm $\|\cdot\|$ defined for each element $\mu$ of $M(G)$ by

$$
\|\mu\|=\operatorname{var}|\mu|
$$

where $|\mu|$ is the element of $M(G)$ defined for each $\mu$-measurable subset $E$ of $G$ by

$$
|\mu|(E)=\text { variation of } \mu \text { on } E \text {, }
$$


(for details, see $\S 19.12$ of [5]). For each element $\psi$ of $L_{1}(G)$ and each element $\mu$ of $M(G)$, let $\mu \omega \psi$ be the function defined for each element $x$ of $G$ by

$$
(\mu \omega \psi\rangle(x)=\int_{G} \psi\left(y^{-1} x\right) \omega\left(y, y^{-1} x\right) d \mu(y) .
$$

The Lebesgue-Fubini Theorem shows that $\mu \omega \psi$ defines an element of $\left(L_{1}(G), \omega\right)$ and a simple computation shows that

$$
\|\mu \omega \psi\|_{1} \leqq\|\mu\|\|\psi\|_{1} \text {. }
$$

Let $W(\mu)$ denote the mapping from $\left(L_{1}(G), \omega\right)$ to itself defined for each element $\psi$ of $L_{1}(G)$ by

We will prove

$$
W(\mu) \psi=\mu \omega \psi \text {. }
$$

Theorem 1. The mapping $\mu \mapsto W(\mu)$ is a linear isometry from $M(G)$ onto $R\left(\left(L_{1}(G), \omega\right)\right)$.

Let $M_{p}(G)$ be the subset of $M(G)$ consisting of point measures. We then prove

Theorem 2. The mapping $\mu \mapsto W(\mu)$ maps $M_{p}(G)$ onto the set of isometries in $R\left(\left(L_{1}(G), \omega\right)\right)$.

Let $G^{\prime}$ be another separable locally compact group, $e^{\prime}$ the unit element in $G^{\prime}, m^{\prime}$ a left-invariant Haar measure on $G^{\prime}$ and $\delta^{\prime}$ the modular function on $G^{\prime}$. Let $\omega^{\prime}$ be an element of $Z^{2}\left(G^{\prime}, T\right)$, let $\varrho$ be an element of $Z^{1}(G, T)$ and let $\tau$ be a topological isomorphism from $G$ onto $G^{\prime}$. (A topological isomorphism between topological groups is an algebraic isomorphism which is also a homeomorphism.) Then, for each element $\psi$ of $L_{1}(G)$, let $\Gamma \psi$ be the element of $L_{1}\left(G^{\prime}\right)$ defined for each element $\tau x$ of $G^{\prime}$ by

$$
(\Gamma \psi)(\tau x)=c(\tau) \varrho(x) \psi(x)
$$

where $c(\tau)$ is the positive real number such that for each m-measurable subset $E$ of $G$

$$
m(E)=c(\tau) m^{\prime}(\tau E) .
$$

[The mapping $E \mapsto m^{\prime}(\tau E)$ is a left-invariant Haar measure on $G$ and so there exists a real constant $c(\tau)$ such that (2.16) holds.]

We will show that, provided that for each pair $x, y$ of elements of $G$,

$$
\varrho(x y)=\varrho(x) \varrho(y) \overline{\omega(x, y)} \omega^{\prime}(\tau x, \tau y),
$$

$\Gamma$ is an isometric *-isomorphism from $\left(L_{1}(G), \omega\right)$ onto $\left(L_{1}\left(G^{\prime}\right), \omega^{\prime}\right)$. Further, using Theorems 1 and 2 , we will prove

Theorem 3. There exists a norm non-increasing isomorphism from $\left(L_{1}(G), \omega\right)$ onto $\left(L_{1}\left(G^{\prime}\right), \omega^{\prime}\right)$ if and only if there exists an element $\varrho$ of $Z^{1}(G, T)$ and a topological isomorphism between $G$ and $G^{\prime}$ such that for each pair $x, y$ of elements of $G,(2.17)$ holds. In this case $\Gamma$ is defined for each element $\psi$ of $L_{1}(G)$ by $(2.15)$.

An immediate corollary of this theorem is

Corollary 4. Every norm non-increasing isomorphism from $\left(L_{1}(G), \omega\right)$ onto $\left(L_{1}\left(G^{\prime}\right), \omega^{\prime}\right)$ is an isometric $*$-isomorphism. 
Let $\operatorname{Aut}(G)$ denote the group of topological automorphisms of $G$. Then $\operatorname{Aut}(G)$ may be regarded as acting on $Z^{2}\left(G, T^{\prime}\right)$ if for each element $\omega$ of $Z^{2}(G, T)$ and each element $\tau$ of $\operatorname{Aut}(G), \tau \omega$ is defined for each pair $x, y$ of elements of $G$ by,

$$
(\tau \omega)(x, y)=\omega\left(\tau^{-1} x, \tau^{-1} y\right) .
$$

Since $B^{2}(G, T)$ is invariant under the action of $\operatorname{Aut}(G)$, there exists an induced action of $\operatorname{Aut}(G)$ on $H^{2}(G, T)$ defined for each element $[\omega]$ of $H^{2}(G, T)$ and each element $\tau$ of $\operatorname{Aut}(G)$ by

$$
\tau[\omega]=[\tau \omega]
$$

([ $\omega]$ denotes the element of $H^{2}(G, T)$ containing $\left.\omega\right)$. Applying Theorem 3 to the case in which $G$ and $G^{\prime}$ are the same group, we prove

Theorem 5. The number, up to isometric *-isomorphism, of twisted group algebras over $G$ with continuous multipliers is equal to the number of orbits in $H^{2}(G, T)$ under the action of $\operatorname{Aut}(G)$.

\section{\$3. Centralisers on $\left(\boldsymbol{L}_{\mathbf{1}}(G), \omega\right)$}

Proof of Theorem 1. For each element $\mu$ of $M(G)$, the mapping $W(\mu)$ : $\left(L_{1}(G), \omega\right) \rightarrow\left(L_{1}(G), \omega\right)$ defined by $(2.14)$ is clearly linear. Moreover (2.13) shows that it is bounded and

$$
\|W(\mu)\| \leqq\|\mu\| \text {. }
$$

A simple computation shows that for each pair $\psi_{1}, \psi_{2}$ of elements of $L_{1}(G)$,

$$
\mu \omega\left(\psi_{1} \omega \psi_{2}\right)=\left(\mu \omega \psi_{1}\right) \omega \psi_{2}
$$

and hence that $W(\mu)$ is an element of $R\left(\left(L_{1}(G), \omega\right)\right)$.

The mapping $W: \mu \rightarrow W(\mu)$ is clearly linear from $M(G)$ onto $R\left(\left(L_{1}(G), \omega\right)\right)$ and $(3.1)$ shows that it is bounded. I et $\mu$ be an element of $M(G)$ such that for each element $\psi$ of $L_{1}(G)$,

$$
\mu \omega \psi=0 \text {. }
$$

In particular (3.3) holds for each element $\psi$ of the space $C_{00}(G)$ of continuous functions having compact support in $G$. It follows that for each element $x$ of $G$,

$$
\int_{G} \psi\left(y^{-1} x\right) \omega\left(y, y^{-1} x\right) d \mu(y)=0 .
$$

In particular (3.4) holds when $x=e$. Clearly the mapping which sends each element $\psi$ of $O_{00}(G)$ into the function $\psi^{\sim}$ defined for each element $y$ of $G$ by

$$
\psi^{\sim}(y)=\psi\left(y^{-1}\right) \omega\left(y, y^{-1}\right)
$$

is one to one onto $C_{00}(G)$. It follows that for each element $\psi$ of $C_{00}(G)$,

$$
\int_{G} \psi(y) d \mu(y)=0 \text {. }
$$

Let $C_{0}(G)$ be the $B_{a}$ nach space of continuous complex-valued functions on $G$ which take arbitrarily small values outside compact subsets of $G$. 
Then Theorem 14.10 of [5] shows that $M(G)$ may be regarded as the Banach space dual of $C_{0}(G)$. Moreover, $C_{00}(G)$ is dense in $C_{0}(G)$ and so (3.5) shows that $\mu=0$. Hence $W$ maps $M(G)$ one to one into $R\left(\left(L_{1}(G), \omega\right)\right)$.

Let $V$ be an element of $R\left(\left(L_{1}(G), \omega\right)\right)$ and let $\left\{\chi_{i}: i \varepsilon \Lambda\right\}$ be an approximate identity for $\left(L_{1}(G), \omega\right)$. (For the definition of approximate jdentities for Banach $*$-algebras see [2] and for the proof of the existence of such an approximate identity for $\left(L_{1}(G), \omega\right)$ see [8]). For each element $i$ of $\Lambda$ let $\eta_{i}=V \chi_{i}$. Now $\left\{\eta_{i}: i \in \Lambda\right\}$ is a sequence of elements of $L_{1}(G)$ such that

$$
\left\|\eta_{i}\right\|_{1}=\left\|V \chi_{i}\right\|_{1} \leqq\|V\|\left\|\chi_{i}\right\|_{1}=\|V\|
$$

An argument identical to that given in the proof of Theorem 1 of [8] shows that there exists an element $\mu$ of $M(G)$ which is a limit point of the sequence $\left\{\eta_{i}: i \in \Lambda\right\}$ in the weak $*$-topology of $M(G)$ when regarded as the dual of $C_{0}(G)$. It follows from (3.6) that

$$
\|\mu\| \leqq\|V\| \text {. }
$$

Again, following the proof of Theorem 1 of [8] with slight modifications involving the multiplier $\omega$ it may be shown that for each element $\psi$ of $L_{1}(G)$,

and hence that $V=W(\mu)$.

$$
V \psi=\mu \omega \psi
$$

Finally it follows from (3.1) and (3.7) that $\|W(\mu)\|=\|\mu\|$ and hence that $W$ maps $M(G)$ isometrically onto $R\left(\left(L_{1}(G), \omega\right)\right)$.

Before proving Theorem 2, it is useful to consider the image of various subset of $M(G)$ under the mapping $W$. Let $M_{a}(G)$ be the closed subspace of $M(G)$ whose elements are absolutely continuous with respect to $m$. To each element $\mu$ of $M_{a}(G)$ there corresponds a unique element $\phi$ of $L_{1}(G)$ such that $d \mu=\phi d m$ and the mapping $\mu \mapsto \phi$ is an isometry from $M_{a}(G)$ onto $L_{1}(G)$ (for full details see $\S 19.18$ of [5]). It is clear that for each element $\psi$ of $L_{1}(G), W(\mu) \psi=\phi \omega \psi$. We have therefore proved

Corollary 3.1. $W$ maps $M_{a}(G)$ isometrically onto the left-multiplication operators on $\left(L_{1}(G), \omega\right)$.

Let $x$ be an element of $G$ and let $v_{x}$ be the element of $M_{p}(G)$ concentrated on the point $x$. Then, for each element of $L_{1}(G)$,

$$
W\left(v_{x}\right) \psi=\mathscr{L}(x) \psi
$$

where $\mathscr{L}(x) \psi$ is the element of $L_{1}(G)$ defined by

$$
(\mathscr{L}(x) \psi)(y)=\omega\left(x, x^{-1} y\right) \psi\left(x^{-1} y\right) .
$$

It was shown in [4] that the mapping $\mathscr{L}(x)$ is a linear isometry from $L_{1}(G)$ onto itself. $\mathscr{L}(x) \psi$ is said to be the twisted left-translate of $\psi$ by $x$ corresponding to $\omega$ and the mapping $\psi \mapsto \mathscr{L}(x) \psi$ is said to be the twisted left-translation operator by $x$ corresponding to $\omega$. A simple computation shows that for each pair $x, y$ of elements of $G$ and each 
element $\psi$ of $L_{1}(G)$,

$$
\mathscr{L}(x) \mathscr{L}(y) \psi=\omega(x, y) \mathscr{L}(x y) \psi .
$$

It therefore follows from (3.9) that the following result has been proved.

Corollary 3.2. $W$ maps $M_{p}(G)$ isometrically onto the set of twisted left-translation operators on $\left(L_{1}(G), \omega\right)$.

So far we have only concerned ourselves with the norm topology of $R\left(\left(L_{1}(G), \omega\right)\right)$. In the proof of Theorem 3 , several results about the strong topology of $R\left(\left(L_{1}(G), \omega\right)\right)$ are required. In particular, it follows immediately from Theorem 1 that

Corollary 3.3. $R\left(\left(L_{1}(G), \omega\right)\right)$ is closed in the strong topology of $B\left(\left(L_{1}(G), \omega\right)\right)$.

Also by closely following the proof of Theorem 4 of [8] with the usual modifications involving $\omega$, it may be proved that

Corollary 3.4. The set finite linear combinations of twisted left-translation operators is strongly dense in $R\left(\left(L_{1}(G), \omega\right)\right)$.

Proof of Theorem 2. Corollary 3.2 shows that $W$ maps $M_{p}(G)$ isometrically into the set of jsometries in $R\left(\left(L_{1}(G), \omega\right)\right)$. Let $V$ be an isometric element of $R\left(\left(L_{1}(G), \omega\right)\right)$ and let $\mu$ be the unique element of $M(G)$ such that $W(\mu)=V$. Then, using the fact that the mapping $\psi \mapsto \psi^{\sim}$ defined by (3.5) maps $C_{00}(G)$ isometrically onto itself, a slight modification of the proof of Theorem 3 of [8] shows that $\mu$ is an element of $M_{p}(G)$.

The detailed proofs of the results of this section are given in [3].

\section{$\$ 4$. Isomorphisms of Twisted Group Algebras}

Proof of Theorem 3. Let $\tau$ be a topological isomorphism from $G$ onto $G^{\prime}$ and let $\varrho$ be an element of $Z^{1}(G, T)$ such that for each pair $x, y$ of elements of $G$,

$$
\varrho(x y)=\varrho(x) \varrho(y) \overline{\omega(x, y)} \omega^{\prime}(\tau x, \tau y),
$$

For each element $\psi$ of $L_{1}(G)$ let $\Gamma \psi$ be the function defined for each element $\tau x$ of $G^{\prime}$ by

$$
(\Gamma \psi)(\tau x)=c(\tau) \varrho(x) \psi(x) .
$$

Since $\varrho$ is continuous, $\Gamma \psi$ is $m$-measurable and

$$
\int_{G}|(\Gamma \psi)(\tau x)| d m^{\prime}(\tau x)=\int_{G} c(\tau)|\varrho(x) \psi(x)| c(\tau)^{-1} d m(x)=\|\psi\|_{1} .
$$

Hence $\Gamma \psi$ defines an element of $L_{1}\left(G^{\prime}\right) . \Gamma$ is clearly a linear mapping from $L_{1}(G)$ into $L_{1}\left(G^{\prime}\right)$ which $(4.3)$ shows to be isometric. Let $\psi^{\prime}$ be an element of $L_{1}\left(G^{\prime}\right)$ and let $\psi$ be the function defined for each element $\tau^{-1} x^{\prime}$ of $G$ by

$$
\psi\left(\tau^{-1} x^{\prime}\right)=c(\tau)^{-1} \varrho\left(\tau^{-1} x^{\prime}\right)^{-1} \psi^{\prime}\left(x^{\prime}\right) .
$$

Then $\psi$ defines an element of $L_{1}(G)$ such that $\Gamma \psi=\psi^{\prime}$. Hence $\Gamma$ is a linear isometry onto $L_{1}\left(G^{\prime}\right)$. Using (4.1) a simple computation shows 
that $\Gamma$ is a homomorphism. For each $m$-measurable subset $E$ of $G$ and each element $x$ of $G$,

$$
\begin{aligned}
\delta^{\prime}(\tau x) m^{\prime}(\tau E)=m^{\prime}(\tau E x) & =c(\tau) m(E x)=\delta(x) c(\tau) m(E) \\
& =\delta(x) m^{\prime}(\tau E)
\end{aligned}
$$

and so for each element $x$ of $G$,

$$
\delta^{\prime}(\tau x)=\delta(x) \text {. }
$$

Using (4.1) and (4.6) a simple computation shows that for each element $\psi$ of $L_{1}(G), \Gamma\left(\psi^{\omega}\right)=(\Gamma \psi)^{\omega^{\prime}}$ from which it follows that $\Gamma$ is an isometric *-isomorphism.

Conversely, let $\Gamma$ be a norm non-increasing isomorphism from $\left(L_{1}(G), \omega\right)$ onto $\left(L_{1}\left(G^{\prime}\right), \omega^{\prime}\right)$ and let $V$ be an element of $R\left(\left(L_{1}(G), \omega\right)\right)$. Then $\Gamma V \Gamma^{-1}$ is clearly an element of $R\left(\left(L_{1}\left(G^{\prime}\right), \omega^{\prime}\right)\right)$. It follows that for each element $z$ of $G, V(z)=\Gamma \mathscr{L}(z) \Gamma^{-1}$ is an element of $R\left(\left(L_{1}\left(G^{\prime}\right), \omega^{\prime}\right)\right)$. Using the same argument as that used in the proof of Lemma 1 of [8] it follows that for each element $\psi^{\prime}$ of $L_{1}\left(G^{\prime}\right)$,

$$
\left\|V(z) \psi^{\prime}\right\|_{1} \leqq\left\|\psi^{\prime}\right\|_{1} .
$$

However, it follows from (3.11) that

$$
V(z)^{-1}=\overline{\omega\left(z, z^{-1}\right)} V\left(z^{-1}\right) .
$$

Replacing $z$ by $z^{-1}$ in (4.7) it follows that $V(z)$ and $V(z)^{-1}$ are both norm non-increasing and therefore isometric. It follows from Theorem 2 that there exist mappings $\tau$ from $G$ to $G^{\prime}$ and $\varrho$ from $G$ to $T$ such that for each element $z$ of $G$,

$$
V(z)=\Gamma \mathscr{L}(z) \Gamma^{-1}=\varrho(z) \mathscr{L}^{\prime}(\tau z)
$$

where $\mathscr{L}^{\prime}\left(x^{\prime}\right)$ denotes the twisted left-translation operator on $L_{1}\left(G^{\prime}\right)$ corresponding to the element $x^{\prime}$ of $G^{\prime}$. Let $x, y$ be elements of $G$. Then, using (3.11) and (4.9),

$$
\begin{aligned}
\varrho(x y) \mathscr{L}^{\prime}(\tau x y) & =\Gamma \mathscr{L}(x y) \Gamma^{-1}=\overline{\omega(x, y)} \Gamma \mathscr{L}(x) \Gamma^{-1} \Gamma \mathscr{L}(y) \Gamma^{-1} \\
& =\overline{\omega(x, y)} \varrho(x) \varrho(y) \mathscr{L}^{\prime}(\tau x) \mathscr{L}^{\prime}(\tau y) \\
& =\overline{\omega(x, y)} \varrho(x) \varrho(y) \omega^{\prime}(\tau x, \tau y) \mathscr{L}^{\prime}(\tau x, \tau y) .
\end{aligned}
$$

It follows that for each element $\psi^{\prime}$ of $L_{1}\left(G^{\prime}\right)$ and $m^{\prime}$-almost every element $z^{\prime}$ of $G^{\prime}$,

$$
\begin{aligned}
\psi^{\prime}\left(z^{\prime}\right) & =\overline{\varrho(x y)} \varrho(x) \varrho(y) \overline{\omega(x, y)} \omega^{\prime}(\tau x, \tau y) \\
& \cdot\left(\mathscr{L}^{\prime}(\tau x y)^{-1} \mathscr{L}^{\prime}(\tau x \tau y) \psi^{\prime}\right)\left(z^{\prime}\right) \\
& =f(x, y) \mathscr{L}^{\prime}\left(\left(\tau y^{-1}(\tau x)^{-1}(\tau x y)\right) \psi^{\prime}\right)\left(z^{\prime}\right),
\end{aligned}
$$

where $f$ is a continuous function from $G \times G$ to $T$. Therefore,

$$
\begin{aligned}
\int_{G^{\prime}} \psi^{\prime}\left(z^{\prime}\right) d m^{\prime}\left(z^{\prime}\right) & =f(x, y) \int_{G^{\prime}} \psi^{\prime}\left((\tau y)^{-1}(\tau x)^{-1}(\tau x y) z^{\prime}\right) d m^{\prime}\left(z^{\prime}\right) \\
& =f(x, y) \int_{G^{\prime}} \psi^{\prime}\left(z^{\prime}\right) d m^{\prime}\left(z^{\prime}\right)
\end{aligned}
$$


from which it follows that $f(x, y)=1$ and hence from (4.12) that for each element $\psi^{\prime}$ of $L_{1}\left(G^{\prime}\right)$ and $m^{\prime}$-almost every element $z^{\prime}$ of $G^{\prime}$,

$$
\psi^{\prime}\left(z^{\prime}\right)=\psi^{\prime}\left((\tau y)^{-1}(\tau x)^{-1} \tau(x y) z^{\prime}\right) .
$$

In particular (4.13) holds for each element $\psi^{\prime}$ of $C_{00}\left(G^{\prime}\right)$. In this case both sides of (4.13) are continuous functions of $z^{\prime}$ and so (4.13) holds for every element $z^{\prime}$ of $G^{\prime}$ and in particular when $z^{\prime}=e^{\prime}$. Since $C_{00}\left(G^{\prime}\right)$ separates points in $G^{\prime}$ it follows that

$$
\tau x y=\tau x \tau y .
$$

Hence $\tau$ is a homomorphism from $G$ to $G^{\prime}$. It therefore follows from (4.10) that $\varrho(e)=1$ and that for each pair $x, y$ of elements of $G,(2.17)$ holds. It must be proved that $\varrho$ is continuous and that $\tau$ is a topological isomorphism. Let $x$ be an element of $G$ such that $\tau x=e^{\prime}$. It follows from (4.9) that for each element $\psi$ of $L_{1}(G)$,

$$
\mathscr{L}(x) \psi=\varrho(x) \psi
$$

and a similar argument to that above shows that $x=e$.

In order to prove the continuity of $\varrho$ and $\tau$ the following Lemma whose proof will be given at the end of the present proof, is required.

Lemma. The mapping $x \mapsto \mathscr{L}(x)$ is continuous in the strong topology of $B\left(L_{1}(G)\right)$.

Continuing the proof of Theorem 3, it is clear that the mapping $\tau$ is composed of the three mappings

$$
\begin{aligned}
& \tau_{1}: x \rightarrow \mathscr{L}(x), \\
& \tau_{2}: \mathscr{L}(x) \rightarrow \Gamma \mathscr{L}(x) \Gamma^{-1}, \\
& \tau_{3}: \mathscr{L}^{\prime}\left(x^{\prime}\right) \rightarrow x^{\prime} .
\end{aligned}
$$

Arguments similar to those used in the proof of Lemma 2 of [8] show $\tau_{2}$ and $\tau_{3}$ to be strongly continuous and the lemma above shows that $\tau_{1}$ is strongly continuous. Hence $\tau$ is continuous from $G$ into $G^{\prime}$. Moreover, for each element $x$ of $G$ and each element $\psi^{\prime}$ of $L_{1}\left(G^{\prime}\right)$,

$$
\varrho(x) \psi^{\prime}=\omega^{\prime}\left(\tau x, \tau x^{-1}\right)\left(\Gamma \mathscr{L}(x) \Gamma^{-1} \mathscr{L}^{\prime}\left(\tau x^{-1}\right) \psi^{\prime}\right)
$$

and so the continuity of $\tau$ shows that $\varrho$ is continuous. Arguments similar to those used in the proof of Lemma 3 of [8] and Corollary 3.4 show that $\tau G=G^{\prime}$.

It remains to prove that $\Gamma$ has the form (2.15). Let $\Gamma^{\prime}$ be the isometric $*$-isomorphism from $\left(L_{1}(G), \omega\right)$ onto $\left(L_{1}\left(G^{\prime}\right), \omega^{\prime}\right)$ defined for each element $\tau x$ of $G^{\prime}$ by

$$
\left(\Gamma^{\prime} \psi\right)(\tau x)=c(\tau) \varrho(x) \psi(x) .
$$

Then a simple computation shows that for each element $y$ of $G$,

$$
\Gamma^{\prime} \mathscr{L}(y) \Gamma^{\prime-1}=\varrho(y) \mathscr{L}^{\prime}(\tau y) .
$$


Let $\Gamma^{\prime \prime}=\Gamma^{-1} \Gamma^{\prime}$. Then $\Gamma^{\prime \prime}$ is a bounded automorphism of $\left(L_{1}(G), \omega\right)$ such that for each element $y$ of $G$,

$$
\Gamma^{\prime \prime} \mathscr{L}(y) \Gamma^{\prime \prime-1}=\mathscr{L}(y) .
$$

A simple limit argument using Corollary 3.4 shows that for each element $V$ of $R\left(\left(L_{1}(G), \omega\right)\right)$.

$$
\Gamma^{\prime \prime} V \Gamma^{\prime \prime-1}=V \text {. }
$$

In particular Corollary 3.1 shows that (4.19) holds when $V$ is the leftmultiplication operator defined by an element $\psi$ of $L_{1}(G)$. Hence, for each pair $\psi, \psi_{1}$ of elements of $L_{1}(G)$,

$$
\psi \omega \Gamma^{\prime \prime} \psi_{1}=\Gamma^{\prime \prime}\left(\psi \omega \psi_{1}\right)=\Gamma^{\prime \prime} \psi \omega \Gamma^{\prime \prime} \psi_{1} .
$$

It follows that for each pair $\psi, \psi_{2}$ of elements of $L_{1}(G)$,

$$
\Gamma^{\prime \prime} \psi \omega \psi_{2}=\psi \omega \psi_{2} \text {. }
$$

Let $\left\{\chi_{i}: i \in \Lambda\right\}$ be an approximate identity for $\left(L_{1}(G), \omega\right)$. Then, for each element $\psi$ of $L_{1}(G)$,

$\left\|\Gamma^{\prime \prime} \psi-\psi\right\|, \leqq\left\|\Gamma^{\prime \prime} \psi-\Gamma^{\prime \prime} \psi \omega \chi_{i}\right\|_{1}+\left\|\Gamma^{\prime \prime} \psi \omega \chi_{i}-\psi \omega \chi_{i}\right\|_{1}+\left\|\psi \omega \chi_{1}-\psi\right\|_{1}$.

It follows from $(4.20)$ that $\Gamma^{\prime \prime} \psi=\psi$ and hence that $\Gamma=\Gamma^{\prime}$. This completes the proof of the theorem.

Proof of Lemma. Let $\psi$ be an element of $L_{1}(G)$ and let $x, y$ be elements of $G$. Then, by (3.11),

$$
\begin{aligned}
\|\mathscr{L}(x y) \psi-\mathscr{L}(x) \psi\|_{1} & =\|\omega(x, y) \mathscr{L}(x) \mathscr{L}(y) \psi-\mathscr{L}(x) \psi\|_{1} \\
& \leqq|\omega(x, y)-1|\|\psi\|_{1}+\|\mathscr{L}(y) \psi-\psi\|_{1},
\end{aligned}
$$

since $\mathscr{L}(x)$ is isometric. It follows that the continuity of the mapping $x \mapsto \mathscr{L}(x) \psi$ at $e$ implies its continuity at every point $y$ of $G$.

Let $\varepsilon>0$ and let $\phi$ be a continuous function of compact support $F$ in $G$ such that

$$
\|\psi-\phi\|_{1}<\varepsilon / 4 \text {. }
$$

Such functions exist since $C_{00}(G)$ is dense in $L_{1}(G)$. Since $\omega$ is continuous on $G \times G$, there exists a neighbourhood $E_{1}$ of $e$ such that

$$
\left|\omega\left(x, x^{-1} y\right)-1\right| \leqq\left(\varepsilon / 4\|\phi\|_{1}\right)
$$

for each element $y$ of $F$ and each element $x$ of $E_{1}$. Therefore,

$$
\begin{aligned}
& \|\mathscr{L}(x) \psi-\psi\|_{1} \leqq\|\mathscr{L}(x) \psi-\mathscr{L}(x) \phi\|_{1}+\|\mathscr{L}(x) \phi-\phi\|_{1} \\
& \quad+\|\phi-\psi\|_{1}<\varepsilon / 2+\int_{G}\left|\phi\left(x^{-1} y\right)-\phi(y)\right| d m(y) \\
& \quad+\int_{G}\left|\omega\left(x, x^{-1} y\right)-1\right||\phi(y)| d m(y) .
\end{aligned}
$$

It follows from $\S 20.4$ of [5] that there exists a neighbourhood $E_{2}$ of $e$ such that for each element $x$ of $E_{2}$,

$$
\int_{G}\left|\phi\left(x^{-1} y\right)-\phi(y)\right| d m(y)<\varepsilon / 4 \text {. }
$$

It follows that the mapping $x \mapsto \mathscr{L}(x) \psi$ is continuous at $e$. 
Proof of Theorem 5. Let $\left(L_{1}(G), \omega_{1}\right),\left(L_{1}(G), \omega_{2}\right)$ be isometrically *-isomorphic twisted group algebras over $G$. Then, applying Theorem 4 when $G=G^{\prime}$, there exist elements $\varrho$ of $Z^{1}(G, T)$ and $\tau$ of $\operatorname{Aut}(G)$ such that for each pair $x, y$ of elements of $G$,

$$
\omega_{1}(x, y)=\varrho(x) \varrho(y) \varrho(x y)^{-1} \omega_{2}(\tau x, \tau y)
$$

which implies that

$$
\omega_{1}=\partial \varrho+\tau^{-1} \omega_{2} .
$$

It follows that $\left[\omega_{2}\right]=\tau\left[\omega_{1}\right]$ and hence that $\left[\omega_{2}\right],\left[\omega_{1}\right]$ are in the same orbit of $H^{2}(G, T)$ under the action of $\operatorname{Aut}(G)$.

Conversely, let $\left[\omega_{1}\right],\left[\omega_{2}\right]$ be elements of $H^{2}(G, T)$ in the same orbit under the action of $\operatorname{Aut}(G)$. Then there exist elements $\varrho$ of $Z^{1}(G, T)$ and $\tau$ of $\operatorname{Aut}(G)$ such that (4.27) holds. It follows that the twisted group algebras $\left(L_{1}(G), \omega_{1}\right),\left(L_{1}(G), \omega_{2}\right)$ are isometrically $*$-isomorphic. This completes the proof of the Theorem.

\section{$\S 5$. Example}

Let $G$ be a connected, simply connected nilpotent Lie group. Then, using techniques of HochschILD [6] and BARGMANN [1] Theorem 5 can be extended to show that up to isometric *-isomorphism the number of twisted group algebras over $G$ is equal to the number of orbits in a 2 -cohomology group of the Lie algebra $g$ of $G$ with coefficients in a onedimensional real vector space, under the action of $\operatorname{Aut}(G)$ (for details see [3]). In particular when $G=\boldsymbol{R}^{2}$ the direct product of the additive group $\boldsymbol{R}$ of real numbers with itself, the theory shows that there are precisely two orbits. These may be represented by the element 1 of $Z^{2}\left(\boldsymbol{R}^{2}, T\right)$ which is equal to 1 at all points of $\boldsymbol{R}^{2} \times \boldsymbol{R}^{2}$ and the element $\omega$ of $Z^{2}\left(\boldsymbol{R}^{2}, T\right)$ which is defined for each pair $x=\left(x_{1}, x_{2}\right), y=\left(y_{1}, y_{2}\right)$ of $\boldsymbol{R}^{2}$ by

$$
\omega(x, y)=\exp \left((i c / 2)\left(x_{1} y_{2}-x_{2} y_{1}\right)\right)
$$

for some real number $c$. In effect therefore there are only two twisted group algebras over $\boldsymbol{R}^{2}$, the group algebra $\left(L_{1}\left(\boldsymbol{R}^{2}\right), *\right)$ where $*$ denotes the usual convolution, and the twisted group algebra $\left(L_{1}\left(\boldsymbol{R}^{2}\right), \omega\right)$.

Von Neumann [7] showed that up to unitary equivalence there is a unique irreducible $*$-representation $\pi$ of $\left(L_{1}\left(\boldsymbol{R}^{2}\right), \omega\right)$ defined on the Hilbert space $L_{2}\left(\boldsymbol{R}^{1}\right)$ for each element $\psi$ of $L_{1}\left(\boldsymbol{R}^{2}\right)$ by

$$
\pi(\psi)=\frac{1}{2 \pi} \int_{\boldsymbol{R}^{2}} \psi\left(x_{1}, x_{2}\right) e^{i c\left(x_{1} Q+x_{2} P\right)} d x_{1} d x_{2}
$$

where $e^{i c\left(x_{1} P+x_{2} Q\right)}$ is the unitary operator defined for each element $\phi$ of $L_{2}\left(\boldsymbol{R}^{1}\right)$ and each element $s$ of $\boldsymbol{R}$ by

$$
\left(e^{i c\left(x_{1} Q+x_{2} P\right)} \phi\right)(s)=e^{i x_{2}\left(s+x_{1} / 2\right)} \phi\left(s+x_{1}\right) .
$$


When $c$ is replaced by $2 \pi / h$ where $h$ is Planck's constant this is the well-known "Weyl mapping" [9]. When $\psi$ is the Fourier transform of a real-valued function on the classical phase space $\boldsymbol{R}^{2}$ of a system with one degree of freedom, Weyl proposed that $\pi(\psi)$ be the self-adjoint operator representing the corresponding quantum-mechanical observable.

Every irreducible *-representation $\pi$ of $\left(L_{1}\left(\boldsymbol{R}^{2}\right), *\right)$ is of the form

$$
\pi(\psi)=\psi^{\sim}\left(a_{1}, a_{2}\right)
$$

where $\psi^{\sim}$ denotes the inverse Fourior transform of $\psi$ and $\left(a_{1}, a_{2}\right)$ is a point in $\boldsymbol{R}^{2}$.

It follows from our results that there are only two possible ways of quantizing a classical system with one-degree of freedom, by WEYL's method. The first gives the usual "Weyl mapping" (5.2) whilst the second associates with each classical observable its value at a point of phase space and in this sense reverts to the classical situation.

\section{References}

1. BargmanN, V.: On unitary ray representations of continuous groups. Ann. Math. 59, 1-46 (1954).

2. Dixmier, J.: Les $C^{*}$-algebras and leurs representations. Paris: Gauthiers-Villars 1964.

3. EDwards, C. M.: Twisted group algebras and their representations. Oxford D. Phil. Thesis (1966).

4. - , and J. T. LewIs: Twisted group algebras I. Commun. math. Phys. 13, 119-130 (1969).

5. Hewite, E., and K. A. Ross: Abstract harmonic analysis I. Berlin-GöttingenHeidelberg: Springer 1963.

6. Hochschild, G.: Group extensions of Lie groups I, Ann. Math. 54, 96-109 (1951).

7. von Neumann, J.: Die Eindeutigkeit der Schrödingerschen Operatoren. Math. Ann. 104, 570-578 (1931).

8. Wendel, J. G.: Left centralisers and isomorphisms of group algebras. Pacific J. Math. 2, 251-261 (1952).

9. Weyc, H.: Theory of groups and quantum mechanics. London: Dover 1931.

C. M. EDwards

The Queen's College

Oxford, G. B.
J. T. Lewis

Brasenose College

Oxford, G. B. 